\title{
Interacción de las Instituciones de Educación Superior con el entorno: Orientaciones, Finalidades y Estrategias. Una revisión sistemática de la literatura, 2017-2021
}

\author{
Interaction of Higher Education Institutions with the environment: Orientations, Purpo- \\ ses and Strategies. A Systematic Literature Review, 2017-2021
}

José Jovanny, Jiménez $\bowtie$

Universidad de Deusto

jovanny.jimenez@opendeusto.es

Bilbao, España

Concepción, Maiztegui-Oñate

Universidad de Deusto

Bilbao, España

José Luís, Da Silva Pinto

Universidad Católica Andrés Bello

Caracas, Venezuela

$\bowtie$ Autor por correspondencia

Cómo referenciar:

Jiménez, J.J., Maiztegui-Oñate, C., \& Da Silva Pinto , J.

L.(2022).

Interacción de las Instituciones de Educación Superior con

el entorno: Orientaciones, Finalidades y Estrategias. Una

revisión sistemática de la literatura, 2017-2021.

Emerging Trends in Education, (4)8A, 62-79

https://doi.org/10.19136/etie.a4n8A.4781

Disponible en:

https://revistas.ujat.mx/index.php/emerging

DOI:

https://doi.org/10.19136/etie.a4n8A.4781

Recibido:

Aceptado:

06/10/2021

$14 / 12 / 2021$

Publicado:

05/01/2022

Emerging Trends in Education

e ISSN: 2594-2840

Volumen 4, Número 8A, Enero - Junio 2022

\section{Resumen:}

La incidencia social de las Instituciones de Educación Superior (IES) asume los planteamientos de la Organización de las Naciones Unidas para la Educación, la Ciencia y la Cultura (UNESCO) de promover el bien común en la sociedad. Esto ha generado algunos debates y artículos científicos con diversas interpretaciones: Responsabilidad social universitaria (RSU), Compromiso universidad-comunidad (CUC) y Compromiso cívico (CC). Por tal razón, es relevante analizar los procesos desarrollados por las IES desde dichos planteamientos. Por ello, se realizó una revisión sistemática donde se revisaron 53 artículos publicados entre el 2017 y el 2021 provenientes de cinco bases de datos, para contrastar las orientaciones, finalidades y estrategias implementadas por las IES en diferentes contextos geográficos y culturales. Los resultados muestran que las IES comparten finalidades como: la promoción de comportamientos éticos y socialmente responsables, la promoción de valores democráticos, la promoción del desarrollo sostenible, el aprendizaje a lo largo de la vida y la promoción de habilidades para mejorar la empleabilidad. Asimismo, se concluye que la RSU, es la orientación cada vez más elegida por las IES para relacionarse con el entorno, por integrar los procesos de gestión, participación con las funciones sustantivas de la educación superior, interdependencia que busca crear impactos positivos a nivel personal y colectivo, en el horizonte del bien común expresados en comportamientos ciudadanos con criterios éticos y responsables.

Palabras clave: Instituciones de educación superior; Bien común; Responsabilidad social universitaria; Compromiso universidadcomunidad; Compromiso cívico.

\begin{abstract}
:
The social impact of Higher Education Institutions (HEIs) assumes the United Nations Educational, Scientific and Cultural Organisation (UNESCO) approach of promoting the common good in society. This has generated some debates and scientific articles with different interpretations: University Social Responsibility (USR), UniversityCommunity Commitment (UCC) and Civic Commitment (CC). For this reason, it is relevant to analyze the processes developed by HEIs from these approaches. Therefore, a systematic review was conducted in which 53 articles from five Spanish and English language databases published between 2017 and 2021 were reviewed in order to contrast the orientations, purposes and strategies implemented by HEIs in different geographical and cultural contexts. The results show that HEIs share goals such as: the promotion of ethical and socially responsible behaviour, the promotion of democratic values, the promotion of sustainable development, lifelong learning and the promotion of skills to enhance employability. It is also concluded that USR is the orientation increasingly chosen by HEIs to relate to the environment, by integrating the processes of management, participation, with the substantive functions of higher education, an interdependence that seeks to create positive impacts at personal and collective level, in the horizon of the common good expressed in citizen behaviours with ethical and responsible criteria.
\end{abstract}

Keywords: Higher education institutions; Common good; University social responsibility; University-community engagement; Civic engagement.

Pag. 62-79 


\section{Interacción de las Instituciones de Educación Superior con el entorno: Orientaciones, Finalidades y Estrategias. Una revisión sistemática de la literatura, 2017-2021}

\section{| Introducción}

La UNESCO (Organización de las Naciones Unidas para la Educación, la Ciencia y la Cultura [UNESCO], 1998, 2015), recomienda a las instituciones de educación superior (en adelante IES) promover relaciones sociales más solidarias que susciten el desarrollo económico, social y cultural. Bajo dicho contexto, diversos autores analizan la relación universidad-sociedad (Farnell et al., 2020; Hoyt, 2014; Preece, 2016; Vallaeys, 2021), para examinar el valor y la relevancia social de las IES (De Ketele, 2008). Aunado a esto, están las contradicciones internas sobre rol de las IES en el desarrollo integral de la sociedad, lo que puede ser visto como una crisis de identidad, o como el paso del "servicio" al "compromiso y la responsabilidad social" (Farnell et al., 2020; Hoyt, 2014; Preece, 2016).

La expectativa es que las IES puedan profundizar la integración transversal del impacto individual y colectivo con la responsabilidad social, tomando en consideración los problemas de desarrollo local, regional y nacional, junto con el desarrollo integral de las personas, adaptando la enseñanza y la investigación para afrontar "la insostenibilidad" de la sociedad actual y los urgentes “desafíos globales” (Farnell et al., 2020; Vallaeys, 2021).

En la literatura científica hay variedad de términos para conceptualizar la relevancia social de las IES y la interacción con el entorno. Una tipología útil fue elaborada por Hollister (2017). La propuesta general de este autor tiene que ver con la elección de enfoques. Algunos casos requieren una mayor correspondencia desde la gerencia hacia las necesidades y expectativas sociales, como la Responsabilidad Social Universitaria ( en adelante RSU); el Compromiso Universidad-Comunidad (en adelante CUC), centrado en la comunidad, para impulsar procesos y estrategias de cambio más locales. finalmente, hay enfoques más relacionados con la participación política como el Compromiso Cívico (en adelante CC). De acuerdo con los perfiles institucionales de las IES, prevalecen algunos enfoques sobre otros, para describir las relaciones con el entorno y su impacto en la sociedad. Para Hollister (2017), estos enfoques no son excluyentes unos de otros y pueden coexistir mutuamente.

Por este motivo, esta investigación busca contrastar las principales finalidades, orientaciones y estrategias en la interacción de las IES con su entorno, empleando una revisión sistemática de literatura científica bajo los parámetros metodológicos de Newman y Gough (2020), a partir de 53 artículos seleccionados de los 293 estudios extraídos de cinco bases de datos, publicados entre el 2017 y el 2021. Este trabajo se suma a la reflexión sobre la interacción de las IES con el entorno como forma de aproximarse a la conceptualización de la educación superior como bien común.

\section{Las relaciones de las IES con el entorno}

En principio, las universidades surgieron como organizaciones académicas para asistir a la sociedad (Benneworth et al., 2018), finalidad heredada por las 
IES. De esta manera, la relación con el entorno es su elemento central. Como toda relación, hay momentos de tensión; Particularmente en las IES, las tensiones se dan entre lo que desean ser frente a lo que creen ser, o lo que son y hacen, como también a las expectativas del entorno (Esteruelas, 2018). Así, los principales análisis de esta relación se hacen desde dos perspectivas: la primera, relacionada con la mirada instrumental, centrada en el potencial de las IES para impulsar el crecimiento económico; mientras que la segunda visión se sitúa en el plano de la responsabilidad cívico-social, donde las IES están "fuertemente comprometidas" al logro del bienestar de toda la sociedad (Medina et al., 2017).

Esta investigación privilegia la segunda perspectiva que, de acuerdo con Locatelli, (2018) es una invitación abierta a las IES a integrar las dimensiones sociales individuales con el bienestar colectivo, a través de la docencia y la investigación. En este sentido, la tendencia es potenciar "el diálogo" con el entorno, para atender las demandas de la sociedad (Farnell et al., 2020; Hollister, 2017; Preece, 2016; Vallaeys, 2021). Por ello, recientemente se han realizado revisiones sistemáticas sobre responsabilidad social universitaria (RSU) y Compromiso UniversidadComunidad (CUC).

En ese orden de ideas, Shek et al. (2017) hicieron una revisión sistemática sobre la RSUy la calidad de vida. Como resultado, los autores recomendaron intensificar el trabajo evaluativo en relación con estas iniciativas. Asimismo, aconsejaron promover iniciativas interinstitucionales, y afirmaron que es necesario reforzar el marco teórico y la investigación básica sobre el tema.

Por otra parte, Benneworth et al. (2018), tras una revisión sistemática y un mapeo critico de la literatura, desarrollaron un enfoque denominado "compromiso universitario-comunidad", definido como un "proceso por el que las universidades se comprometen con las partes interesadas de la comunidad para llevar a cabo actividades conjuntas que pueden ser mutuamente beneficiosas, aunque cada parte se beneficie de forma diferente" (p. 17).

Asimismo, se encontraron otras investigaciones afines a la temática realizada por otros autores. Por ejemplo, Martínez-Usarralde et al., (2019), analizan las interacciones entre la RSU y el Aprendizaje-Servicio; la tercera misión y su relación con el crecimiento económico es revisada por Compagnucci y Spigarelli (2020); y Schnurbus y Edvardsson (2020).

En el contexto anterior, este artículo presenta una revisión general que incorpora distintos términos, intentando responder la pregunta ¿Cuáles son las orientaciones, finalidades y estrategias desarrolladas por las IES en diferentes contextos geográficos y culturales descritos en la literatura durante el periodo 2017- 2021?

\section{| Método}

El artículo se enmarca en un enfoque cualitativo, desde una revisión sistemática de carácter exploratorio, que de acuerdo con Jiménez y Aldana (2020) "es un proceso que describe el conocimiento existente de un concepto, temática o problemática particular para dar a conocer el estado investigativo acerca de un problema" (p. 1). La presente revisión se inspiró, inicialmente, en los primeros seis pasos de Prisma (2009), pero eligió la metodología recomendada por Newman y Gough (2020), para sintetizar evidencia sistemáticamente en educación, a partir de un diseño que inicia con la pregunta de investigación, de la cual se obtiene los términos claves para buscar la información y establecer los criterios de inclusión e exclusión, para finalizar con el análisis de los resultados según las categorías establecidas, el cual debe proporcionar una síntesis agregativa de la información. 
Este protocolo, en esta investigación se desarrolló en tres

fases: establecer la pregunta del examen (Primera fase); determinar las palabras claves para la búsqueda en las bases de datos (Segunda fase); y redactar los criterios de inclusión y exclusión (Tercera fase), tal como se observa en la Tabla 1.

Tabla 1

Primera fase de la revisión sistemática

Pregunta para la revisión

Términos claves para la búsqueda

Criterios de Inclusión

Criterios de exclusión

Otros filtros aplicados

Bases de datos:

Web of Science

Scopus

ERIC

SCIELO

ProQuest

Total

Nota: Elaboración propia con base en los registros de inscripción al Diplomado (ambas convocatorias).

La segunda fase fue establecer las categorías que permitieran "analizar, en forma condensada, el conjunto completo de datos, para poder ver literalmente qué hay allí" (Huberman y Miles, 1994, p. 424). Para construir las categorías ("ubicación geográfica, características de los estudios, orientaciones empleadas y estrategias"), se consultaron revisiones sistemáticas relacionadas con el tema o similares (Benneworth et al., 2018; Compagnucci y Spigarelli, 2020; Martínez-Usarralde et al., 2019; Shek et al., 2017; Schnurbus y Edvardsson, 2020).

Para la categoría "finalidades", se toma como referencia "los
¿Cuáles son las orientaciones, finalidades y estrategias desarrolladas por las IES en la interacción con el entorno en los diferentes contextos geográficos y culturales, descritos en la literatura durante el periodo 2017-2021?

Higher education, community-university, engagement, partnerships, social responsability, y civic engagement. En español se emplearon los siguientes términos: Responsabilidad Social Universitaria, extensión y proyección social universitaria.

a)Tipo de documento, eligiendo artículos científicos de revistas indexadas evaluados por pares; b) trabajos académicos originales escritos en inglés o español d)literatura gris proveniente de las tesis doctorales publicadas en ProQuest; e) utilizar el término "universidad" e "instituciones de educación superior" de manera indiferente.

a)Estudios teóricos, revisiones bibliográficas o sistemáticas, informes de políticas; b) se excluyeron artículos fuera del contexto universitario; c) Acceso por suscripción d) bases datos secundarias; e) Otras lenguas.

a)Año: 2017-2021; b) País: cualquiera c) Área: ciencias sociales, d) Operadores booleanos: AND, OR y NOT 


\section{Tabla 2}

Categorías para el análisis

\begin{tabular}{|c|c|}
\hline Categorías & Definición \\
\hline Ubicación geográfica & $\begin{array}{l}\text { Hace referencia al lugar en donde se lleva a cabo el estudio. Responde a la pregunta de la revisión } \\
\text { acerca de los entornos ubicados en diferentes contextos geográficos y culturales }\end{array}$ \\
\hline Características de los estudios & $\begin{array}{l}\text { Refiere a la metodología empleada, el tipo de IES y si hay trabajos que comparan actividades de las } \\
\text { IES en diferentes lugares. Complementa la categoría anterior. }\end{array}$ \\
\hline Orientaciones empleadas & $\begin{array}{l}\text { Se relaciona con "la conceptualización" sobre la que se fundamenta la relaciones de las IES con el } \\
\text { entorno, y la misma sigue la tipología propuesta por Hollister (2017): RSU, CUC y CC. }\end{array}$ \\
\hline Finalidades & $\begin{array}{l}\text { Refiere a los fines perseguidos por las IES en la interacción con el entorno, a partir de las orienta- } \\
\text { ciones halladas. }\end{array}$ \\
\hline Estrategias & $\begin{array}{r}\text { Refieren a las métodos, técnicas y actividades desarrolladas por las IES para lograr los fines. Por } \\
\text { ejemplo, pedagogías empleadas o políticas institucionales desarrolladas. }\end{array}$ \\
\hline
\end{tabular}

Nota: Elaboración propia con base en los registros de inscripción al Diplomado (ambas convocatorias).

El diagrama de flujo presentado en la Figura 1, inspirado en Prisma, (2009), muestra el proceso de cribado de los trabajos en base a los criterios de inclusión-exclusión

\section{Figura 1}

Flujograma de la información de las dos primeras fases de la revisión establecidos. Luego se creó una base datos en Excel, a partir de la cual se hizo el análisis descriptivo y temático.

\section{Inciusión de los estudies analizados}

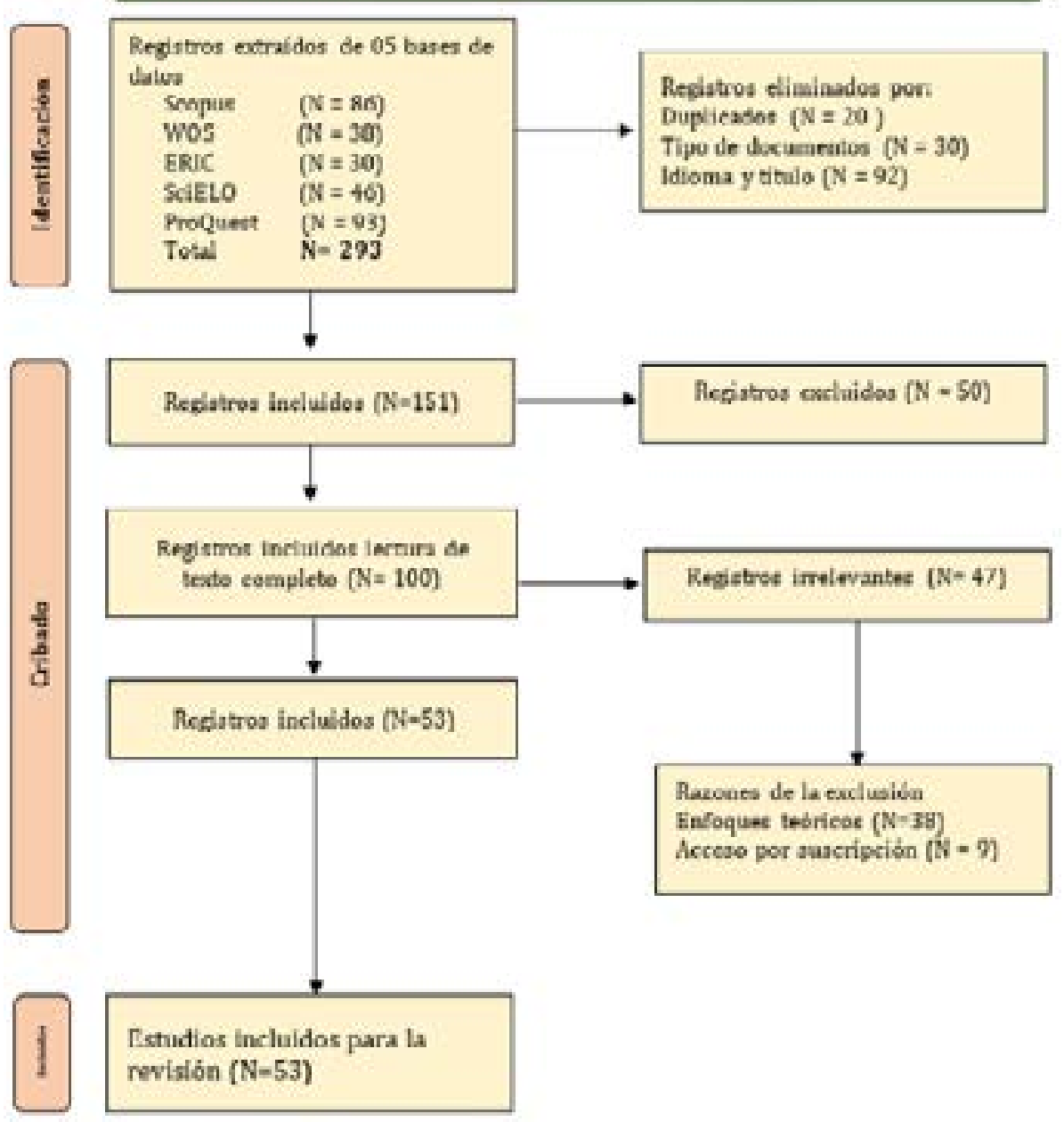


Finalmente, en la tercera fase, se categorizaron todos los registros y se incluyeron ( $N=53)$ artículos presentados en la Tabla 3.

Tabla 3

Listado de artículos seleccionados

\begin{tabular}{|c|c|c|c|}
\hline N. ${ }^{\circ}$ & Autor/es & Título & Año \\
\hline 1 & Alcantar, C. & $\begin{array}{c}\text { Cultivating our nation's engaged citizenry: institutional factors that promo- } \\
\text { te the civic engagement of college students }\end{array}$ & 2017 \\
\hline 2 & Ahumada-Tello et al. & $\begin{array}{l}\text { Responsabilidad Social Universitaria. Desarrollo de competitividad organi- } \\
\text { zacional desde el proceso educativo }\end{array}$ & 2018 \\
\hline 3 & Appe et al. & $\begin{array}{l}\text { The concept and context of the engaged university in the Global South: les- } \\
\text { sons from Latin America to guide a research Agenda }\end{array}$ & 2017 \\
\hline 4 & Bakar et al. & Managing university-community engagement (UCE): The case of UUM & 2019 \\
\hline 5 & Balázs et al. & $\begin{array}{c}\text { Social responsibility and community engagement at a hungarian regional } \\
\text { university }\end{array}$ & 2021 \\
\hline 6 & Bhagwan, R. & $\begin{array}{l}\text { University-community partnerships: demystifying the Process of Engage- } \\
\text { ment }\end{array}$ & 2018 \\
\hline 7 & Bhagwan, R. & $\begin{array}{l}\text { Student volunteer experiences as a way to advance teaching and learning: a } \\
\text { call for community service }\end{array}$ & 2020 \\
\hline 8 & Callan, M. & $\begin{array}{c}\text { An Examination of Benefit and Equity in Community-University Servi- } \\
\text { ce-Learning Partnerships }\end{array}$ & 2020 \\
\hline 9 & Coelho y Menezes. & $\begin{array}{l}\text { University social responsibility as a driving force of change: students' per- } \\
\text { ceptions beyond the ivory tower }\end{array}$ & 2020 \\
\hline 10 & Corretgé y Miret. & $\begin{array}{l}\text { Adaptación de los estándares GRI y creación de indicadores de RSU: un tra- } \\
\text { bajo conjunto de la Universitat de Barcelona y la Universitat Pompeu Fabra }\end{array}$ & 2018 \\
\hline 11 & Cox, J. & $\begin{array}{l}\text { Beyond objectivity: examining the effects of incorporating civic engagement } \\
\text { into higher education journalism courses }\end{array}$ & 2018 \\
\hline 12 & Davis, $\mathrm{M}$. & $\begin{array}{c}\text { Civic engagement of campus-based and online undergraduate students: a } \\
\text { quantitative causal-comparative study }\end{array}$ & 2019 \\
\hline 13 & Dirksen, C. & $\begin{array}{l}\text { Community engagement for student faith development: service-learning in } \\
\text { the Pentecostal tradition }\end{array}$ & 2020 \\
\hline 14 & Dulaney, T. & $\begin{array}{c}\text { How can clarion University of Pennsylvania support the region, employers, } \\
\text { and students in order to meet emerging and changing workforce needs in } \\
\text { the petrochemical and plastics industries }\end{array}$ & 2019 \\
\hline 15 & Douglas et al. & $\begin{array}{c}\text { Embedding tertiary education in rural communities: building 'warm con- } \\
\text { nections' }\end{array}$ & 2020 \\
\hline 16 & Exner et al. & $\begin{array}{l}\text { A Multidisciplinary Approach to University Engagement: Key Considera- } \\
\text { tions for Dynamic Mental Health Teaching and Service Provision to a Disad- } \\
\text { vantaged Community }\end{array}$ & 2017 \\
\hline 17 & Gaete, $\mathrm{R}$. & $\begin{array}{l}\text { Conciliación trabajo-familia y Responsabilidad Social Universitaria: Expe- } \\
\text { riencias de mujeres en cargos directivos en universidades chilenas }\end{array}$ & 2018 \\
\hline 18 & Gaete y Álvarez. & $\begin{array}{l}\text { Responsabilidad social universitaria en Latinoamérica. Los casos de URSU- } \\
\text { LA y AUSJAL }\end{array}$ & 2019 \\
\hline 19 & Gaete, $\mathrm{R}$. & $\begin{array}{c}\text { Dirección por valores y responsabilidad social en universidades estatales } \\
\text { chilenas }\end{array}$ & 2020 \\
\hline 20 & Goldner y Golan. & $\begin{array}{l}\text { What Is Meaningful Civic Engagement for Students? Recollections of Jewish } \\
\text { and Palestinian Graduates in Israel }\end{array}$ & 2019 \\
\hline 21 & Gómez, et al. & $\begin{array}{l}\text { Implementing University Social Responsibility in the Caribbean: Perspecti- } \\
\text { ves of Internal Stakeholders }\end{array}$ & 2018 \\
\hline 22 & González-Pérez et al. & $\begin{array}{c}\text { Crises conducting stakeholder salience shifts in the evolution of private } \\
\text { universities' governance in Latin America }\end{array}$ & 2021 \\
\hline 23 & Gregorova y Heinzova. & $\begin{array}{l}\text { Specifics of Measuring Social and Personal Responsibility of University Stu- } \\
\text { dents after Completion of a Service-Learning Course in Slovak Conditions }\end{array}$ & 2019 \\
\hline 24 & Grobbelaar et. al & $\begin{array}{l}\text { Reinforcing paternalism? the need for a social justice approach to prepare } \\
\text { students for community engagement at universities technology }\end{array}$ & 2017 \\
\hline 25 & Guignona et al. & $\begin{array}{l}\text { A Curriculum for Achieving Universal Health Care: A Case Study of Ateneo } \\
\text { de Zamboanga University School of Medicine }\end{array}$ & 2021 \\
\hline
\end{tabular}




\begin{tabular}{|c|c|c|c|}
\hline 26 & $\begin{array}{l}\text { Huerta-Riveros y Gae- } \\
\text { te-Feres. }\end{array}$ & $\begin{array}{l}\text { Responsabilidad social universitaria a través de los reportes de sostenibili- } \\
\text { dad del Global Reporting Initiative: experiencia de una universidad pública }\end{array}$ & 2017 \\
\hline 27 & Jie y Huam. & $\begin{array}{l}\text { Predictors of reputation through university social responsibility practices in } \\
\text { a Malaysian private university: The customer's perspective }\end{array}$ & 2019 \\
\hline 28 & Joseph y Carolissen. & Citizenship: A core motive for South African university student volunteers & 2019 \\
\hline 29 & Karliani et al. & Indonesian civic engagement among college students & 2019 \\
\hline 30 & Latif, $\mathrm{K}$. & $\begin{array}{l}\text { The Development and Validation of Stakeholder-Based Scale for Measuring } \\
\text { University Social Responsibility (USR) }\end{array}$ & 2018 \\
\hline 31 & Lawton, $\mathrm{P}$. & $\begin{array}{l}\text { At the Crossroads of Intersecting Ideologies: Community-Based Art Educa- } \\
\text { tion, Community Engagement, and Social Practice Art }\end{array}$ & 2019 \\
\hline 32 & Lima-Ravelo et al. & $\begin{array}{c}\text { Impact measuring of university social responsibility. Case university Santo } \\
\text { Tomás, Colombia [Medición del impacto de Responsabilidad Social Univer- } \\
\text { sitaria. Caso Universidad Santo Tomás, Colombia] }\end{array}$ & 2018 \\
\hline 33 & $\begin{array}{l}\text { Mercer-Mapstone y Bo- } \\
\text { vill. }\end{array}$ & $\begin{array}{l}\text { Equity and diversity in institutional approaches to student-staff partners- } \\
\text { hip schemes in higher education }\end{array}$ & 2020 \\
\hline 34 & Maistry y Lortan. & $\begin{array}{l}\text { Lessons from the global south: knowledge democracy and epistemic justice } \\
\text { in higher education institutions in South Africa }\end{array}$ & 2017 \\
\hline 35 & Mbah, M. & $\begin{array}{l}\text { Can local knowledge make the difference? Rethinking universities' commu- } \\
\text { nity engagement and prospect for sustainable community development }\end{array}$ & 2019 \\
\hline 36 & Martínez-Buján et al. & $\begin{array}{l}\text { Campus greening from social sciences: emerging formulas on social respon- } \\
\text { sibility and teaching innovation }\end{array}$ & 2020 \\
\hline 37 & Mbah et al. & $\begin{array}{l}\text { Institutionalizing the intangible through research and engagement: Indi- } \\
\text { genous knowledge and higher education for sustainable development in } \\
\text { Zambia }\end{array}$ & 2021 \\
\hline 38 & Menegat et al. & $\begin{array}{l}\text { Principios de responsabilidad social en la Educación Superior: tendencias } \\
\text { en la gestión de instituciones confesionales }\end{array}$ & 2018 \\
\hline 39 & Mitchell et al. & $\begin{array}{c}\text { Civic Agency and Political Engagement: Community Engagement's Endu- } \\
\text { ring Influence }\end{array}$ & 2019 \\
\hline 40 & Olson, B. & $\begin{array}{l}\text { Higher Education and Community Development: A Multi-stakeholder View } \\
\text { on the Correlates of Project Participation and Design Preferences }\end{array}$ & 2019 \\
\hline 41 & Ossola, M. & $\begin{array}{l}\text { Educación Superior y diversidad cultural: Análisis de las experiencias for- } \\
\text { mativas de jóvenes indígenas en proyectos de extensión universitaria }\end{array}$ & 2018 \\
\hline 42 & $\begin{array}{l}\text { Owusu-Agyeman, } \\
\text { Fourie-Malherbe. }\end{array}$ & $\begin{array}{c}\text { Students as partners in the promotion of civic engagement in higher edu- } \\
\text { cation }\end{array}$ & 2019 \\
\hline 43 & Palombaro et al. & $\begin{array}{c}\text { Assessing the Development of Civic Mindedness in a Cohort of Physical } \\
\text { Therapy Students }\end{array}$ & 2017 \\
\hline 44 & Quillinan et al. & $\begin{array}{l}\text { Lessons learned from a community engagement initiative within Irish hi- } \\
\text { gher education }\end{array}$ & 2018 \\
\hline 45 & Reilly, J. & $\begin{array}{l}\text { A Qualitative Case Study of Engagement in Higher Education: The Influen- } \\
\text { ce of Teachers' Experiences on Classroom Engagement at a Southern State } \\
\text { University }\end{array}$ & 2018 \\
\hline 46 & Karisik, R. & $\begin{array}{l}\text { Community Partners' Perspectives and the Faculty Role in Community-Ba- } \\
\text { sed Learning }\end{array}$ & 2020 \\
\hline
\end{tabular}

Bridging recognition of prior learning (Rpl) and corporate social responsi-

47 Roy y El Marsafawy. bility (csr): Circular flow of interaction among the university, industry, and people

48 Ruiz-Bejarano y Alastor La organización de jornadas en el aula académica: Innovación docente y Compromiso Social Universitario

49 Salcedo-Muñoz et al. Enfoque exploratorio sobre acciones de Responsabilidad Social en universidades del Ecuador: Caso UPSE y UTMACH

Transformation and Development of University Students through Service-Learning: A Corporate-Community-University Partnership Initiative in Hong Kong (Project WeCan)

51 Vásquez, I. La Responsabilidad Social en la Educación Superior: una revisión de la aliinstituciones de educación superior de Oaxaca.

$52 \quad$ Vallaeys y Álvarez.

Hacia una definición Latinoamericana de responsabilidad social universitaria. Aproximación a las preferencias conceptuales de los universitarios 
| Resultados

Una vez incluidos los artículos de la revisión sistemática $(\mathrm{N}=53)$, se procedió a identificar los elementos que daban respuesta a la pregunta de investigación (Tabla 3). Los artículos se analizaron según las categorías descritas en el siguiente análisis.

\section{Ubicación geográfica}

Los resultados muestran que la mayoría de los estudios analizados se han realizado en Estados Unidos, seguido de Sudáfrica, España, Chile, México, Perú, Reino Unido, Malasia, Argentina y Colombia (Figura 2). Asimismo, es posible apreciar la presencia de China a través de Hong Kong o países centroeuropeos como Eslovenia, y de Europa oriental con Hungría.

\section{Figura 2}

Ubicación geográfica

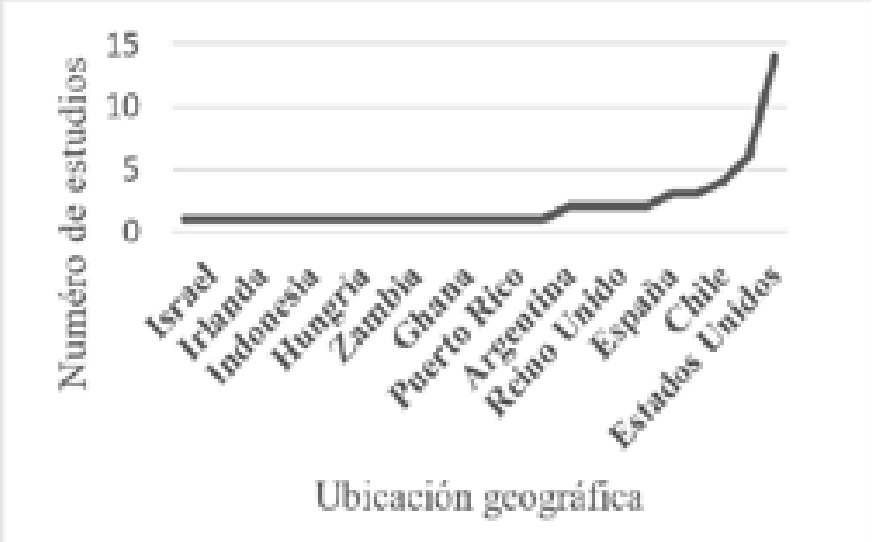

\section{Características de los estudios (Tipos de IES )}

Entre los resultados se evidencia que las IES públicas lideran la reflexión sobre el tema. Entre los 53 trabajos revisados, 36, pertenecían a actividades desarrolladas en diferentes lugares por los sistemas de educación superior pública. Sólo cinco investigaciones, se enfocaban en IES públicas y privadas. Y los 13 trabajos restantes, hacían su análisis en IES privadas. En la Figura 3 se ilustra este hallazgo.

Figura 3

Tipos de IES analizadas en los artículos

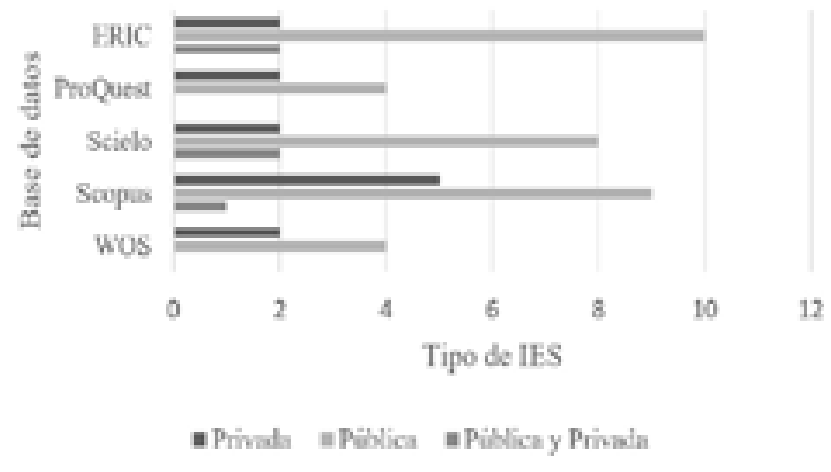

La figura 4, muestra que la metodología más utilizada es estudios de casos. Aunque, otros métodos como investigación-acción y estudios exploratorio descriptivos, también se han empleado. Igualmente, se aprecia que, de los 53 artículos, solo seis trabajos realizaron estudios comparando diferentes IES a nivel nacional (Estados Unidos y España), regional ( Estados unidos y Latinoamérica, dentro de la Unión Europea y entre IES de Latinoamérica) o entre continentes (Estados Unidos y la Unión Europea, Asia y Latinoamérica).

Figura 4

Metodología empleada y estudios comparados

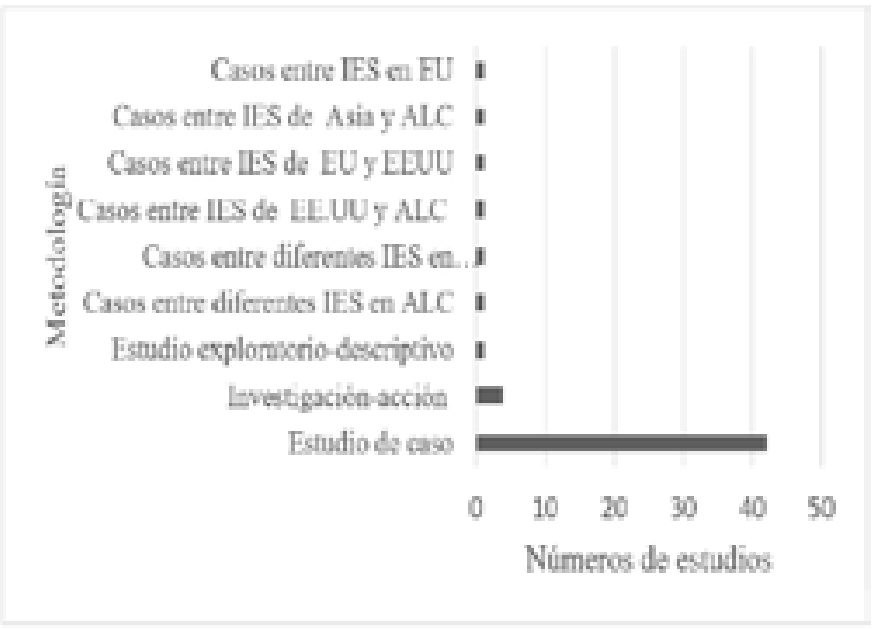


Interacción de las Instituciones de Educación Superior con el entorno: Orientaciones, Finalidades y Estrategias. Una revisión sistemática de la literatura, 2017-2021

\section{Orientaciones en la relación de las IES con el entorno}

En los artículos analizados, se evidencia el predominio de las orientaciones sugeridas por Hollister (2017), RSU, CUC y CC, para interpretar la relación de las IES con el entorno. De los 53 artículos, 21, abordan las actividades desarrolladas a partir de la RSU; seguido de 18 trabajos centrados en el CUC; luego, el CC, representado por ocho estudios; mientras que cuatro investigaciones usan el CUC-RSU, de manera indistinta. Otra conceptualización hallada es la extensión universitaria (en adelante EXTU), con un artículo que reflexiona sobre las experiencias formativas de jóvenes indígenas, en la Universidad de Salta-Argentina (Ossola, 2018); Finalmente, se encuentra la investigación comprometida (en adelante ER), una interpretación señalada por Jiménez, et al. (2021) al analizar el tipo de investigación promovida en algunas redes universitarias. Representado aquí con el trabajo realizado en Zambia sobre los aportes del conocimiento indígena a la educación superior para lograr el desarrollo sostenible (Mbah et al., 2021). La Figura 5 refleja los resultados.

\section{Figura 5}

Orientaciones en la relación de las IES con el entorno

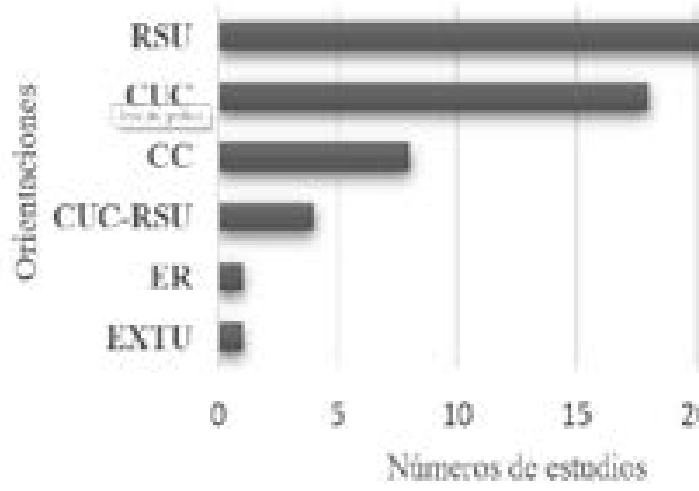

Los resultados evidencian que cada orientación está relacionada con las demandas sociales de los diferentes contextos geográficos y culturales. El CUC, tiene una gran receptividad en Sudáfrica como impulso para la transformación de la sociedad (Bhagwan, 2018, 2020; Exner et al., 2017; Mbah, 2019). El CC, es una tradición arraigada en las IES de EE.UU, para promocionar la democracia, y los valores sociales, (Alcantar, 2017; Grobbelaar et al., 2017; Mitchell et al., 2019), Campus Compact, es un claro ejemplo de ello. La ER, es una propuesta difundida por las IES en Europa, (Jiménez et al., 2021) proyectándose a otros lugares (Mbah et al.,2021). Finalmente, la EXTU y la RSU, es un aporte de Latinoamérica fundamentado en la solidaridad, característica relevante de la misión de las IES. Igualmente, el camino de la EXTU a la RSU refleja la reinterpretación de las relaciones socioeducativas con el entorno, de acuerdo con las demandas sociales del siglo XXI, permitiendo la expansión de esta orientación a diferentes continentes (Ahumada-Tello et al., 2018; Appe et al., 2017; Gaete y Álvarez, 2019; Lima-Ravelo et al., 2018; Martínez-Buján et al., 2020; Ossola, 2018; Shek et al., 2020; Vallaeys y Álvarez, 2019; Yampufé et al., 2021).

\section{Finalidades de la interacción de las IES con el entorno.}

La revisión muestra que entre los artículos que utilizan la orientación de RSU, 11 estudios, tenían como finalidad promover comportamientos éticos y socialmente responsables en la sociedad; siete trabajos eran sobre prácticas relacionadas con el desarrollo sostenible de la sociedad;y cuatro investigaciones, atendían las actividades relacionadas con la promoción de valores democráticos, cívicos y sociales (ciudadanía, participación, justicia social, solidaridad, igualdad, diversidad e inclusión). Mientras que, en el caso del CUC, ocho artículos estaban centrados en el desarrollo sostenible de la sociedad; y en los 14 estudios restantes de esta orientación se encuentran entremezclados la finalidad de promover comportamientos éticos y socialmente responsables en la sociedad con la promoción de valores democráticos, cívicos y sociales. Asimismo, se encontraron dos trabajos que utilizan indistintamente la RSU y el CUC (Tabla 4). 
Además, cinco trabajos trataban exclusivamente de la promoción de valores democráticos, cívicos y sociales, con la orientación del CC. Finalmente, se encontró un artículo relacionado con EXTU cuyo propósito era promover los valores democráticos, cívicos y sociales y un último artículo con la orientación ER, cuya finalidad era promover el desarrollo sostenible de la sociedad.

\section{Estrategias para lograr los fines en la interacción de las IES con}

\section{el entorno}

A partir de esta revisión sistemática, se puede afirmar que distintas orientaciones (RSU, CUC, CC,EXTU y ER), comparten estrategias semejantes en relación con los procesos sustantivos de las IES. Algunas de estas son: Aprendizaje-servicio (ApS), Aprendizaje basado en la comunidad (ABC); Educación para el desarrollo sostenible (EDS);Voluntariado Actualización del currículo; Investigación comprometida; Investigación pertinente; Creación colaborativa del conocimiento. Con relación a las políticas internas de las IES, la gestión socialmente responsable, es una estrategia promovida por la RSU como parte del liderazgo institucional, pero se ha convertido en un elemento común y compartido en las políticas internas de las IES. Finalmente, para la participación social, las estrategias se relacionan con el compromiso del estudiante, compromiso del docente o profesor, y el liderazgo social. La Figura 6 ilustra las diferentes estrategias.

\section{Figura 6}

Estrategias de las IES para interactuar con el entorno

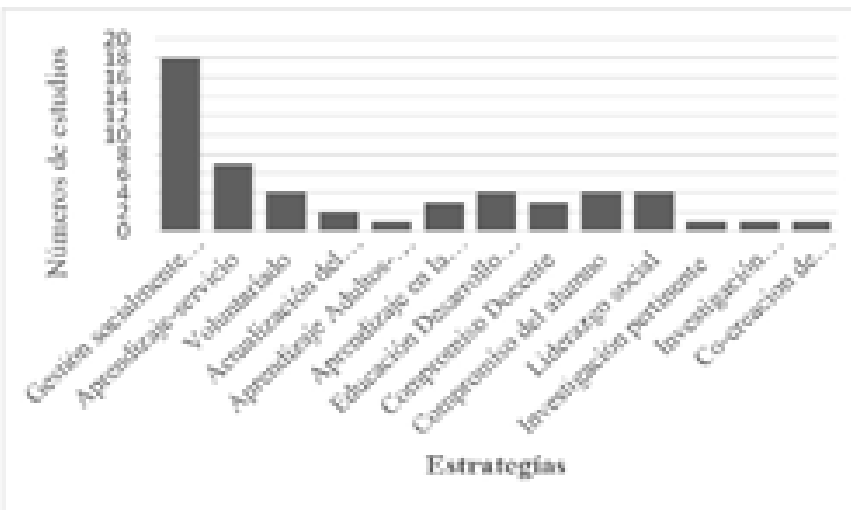

A continuación, la Tabla 4 presenta una síntesis de la relación entre las finalidades, orientaciones, estrategias y autores, en la interacción de las IES con el entorno.

Tabla 4

Relación entre finalidades, orientaciones y estrategias en la interacción de las IES con el entorno

\begin{tabular}{|c|c|c|c|}
\hline Finalidades IES & Orientaciones & Estrategias & Autores \\
\hline \multirow{14}{*}{$\begin{array}{l}\text { Promoción } \\
\text { de comportamientos } \\
\text { éticos y socialmente res- } \\
\text { ponsables en la socie- } \\
\text { dad. }\end{array}$} & \multirow{5}{*}{ RSU } & $\begin{array}{l}\text { Gestión socialmente respon- } \\
\text { sable. }\end{array}$ & \multirow{5}{*}{$\begin{array}{l}\text { Ahumada-Tello et al., 2018; Appe et al., 2017; Coelho y Me- } \\
\text { nezes 2020; Corretgé y Miret, 2018; Dulaney, 2018; Exner et } \\
\text { al., 2017; Gaete, 2018-2020; Gaete y Álvarez, 2019; Gómez et } \\
\text { al., 2018; González-Pérez et al., 2021; Grobbelaar et al., 2017; } \\
\text { Huerta-Riveros y Gaete-Feres, 2017; Latif, 2018; Lima-Ravelo } \\
\text { et al., 2018; Martínez-Buján et al., 2020; Menegat et al., 2018; } \\
\text { Ruiz-Bejarano y Alastor, 2018; Salcedo-Muñoz et al., 2017; } \\
\text { Shek et al., 2020; Vásquez, 2019; Vallaeys y Álvarez, 2019; } \\
\text { Yampufé et al., 2021. }\end{array}$} \\
\hline & & Participación social. & \\
\hline & & $\begin{array}{c}\text { Enseñanza-aprendizaje } \\
\text { (aprendizaje-servicio). }\end{array}$ & \\
\hline & & Compromiso del estudiante. & \\
\hline & & $\begin{array}{c}\text { Investigación útil y pertinen- } \\
\text { te. }\end{array}$ & \\
\hline & \multirow{4}{*}{$\mathrm{CC}$} & $\begin{array}{c}\text { Enseñanza-aprendizaje } \\
\text { (aprendizaje-servicio). }\end{array}$ & \multirow{4}{*}{$\begin{array}{l}\text { Alcantar, 2017; Davis, 2020; Goldner y Golan, 2019; } \\
\text { Karliani et al.,2019; Mitchell et al.,2019; Owusu-Agyeman y } \\
\text { Fourie-Malherbe, 2019; Palombaro et al., } 2017 .\end{array}$} \\
\hline & & Voluntariado. & \\
\hline & & Participación social. & \\
\hline & & Investigación & \\
\hline & \multirow{5}{*}{ CUC } & Participación social. & \multirow{5}{*}{$\begin{array}{l}\text { Bhagwan, 2018-2020; Bakar et al., 2019; Cox, 2018; Dirksen, } \\
\text { 2020; Dulaney, 2018; Exner et al., 2017; Grobbelaar et al., } \\
\text { 2017; Karasik, 2020; Quillinan et al., 2018; Reilly, } 2018 .\end{array}$} \\
\hline & & $\begin{array}{l}\text { Enseñanza-aprendizaje (ApS } \\
\text { y aprendizaje basado a la co- } \\
\text { munidad). }\end{array}$ & \\
\hline & & Voluntariado. & \\
\hline & & Compromiso del profesor. & \\
\hline & & $\begin{array}{c}\text { Creación colaborativa del co- } \\
\text { nocimiento }\end{array}$ & \\
\hline
\end{tabular}


Educación para el desarrollo

CUC sostenible.

\begin{tabular}{|c|c|c|c|}
\hline \multirow{5}{*}{$\begin{array}{l}\text { Promoción del Desarro- } \\
\text { llo sostenible }\end{array}$} & \multirow{3}{*}{$\begin{array}{l}\text { CUC } \\
\text { CC }\end{array}$} & & \multirow{4}{*}{$\begin{array}{l}\text { Alcantar, 2017; Appe et al., 2017; Bhagwan, 2020; Corretgé y } \\
\text { Miret, 2018; Guignona et al., 2021; Lima-Ravelo et al., 2018; } \\
\text { Karasik, 2020; Roy y El Marsafawy, 2021; Owusu-Agyeman } \\
\text { y Fourie-Malherbe, 2019; Vallaeys y Álvarez, 2019; Vallaeys, } \\
\text { 2021. }\end{array}$} \\
\hline & & $\begin{array}{l}\text { Creación colaborativa del co- } \\
\text { nocimiento. }\end{array}$ & \\
\hline & & Participación social & \\
\hline & RSU & $\begin{array}{l}\text { Gestión socialmente respon- } \\
\text { sable. Investigación pertinen- } \\
\text { te. }\end{array}$ & \\
\hline & ER & Investigación comprometida & Mbah et al.,2021. \\
\hline \multirow{10}{*}{$\begin{array}{l}\text { Promoción de valores } \\
\text { democráticos, cívicos } \\
\text { y sociales (ciudadanía, } \\
\text { participación, justicia } \\
\text { social, solidaridad, igual- } \\
\text { dad, diversidad e inclu- } \\
\text { sión) }\end{array}$} & \multirow{4}{*}{ CUC } & $\begin{array}{l}\text { Enseñanza-aprendizaje } \\
\text { (aprendizaje basado en la co- } \\
\text { munidad). }\end{array}$ & \multirow{4}{*}{$\begin{array}{l}\text { Bhagwan, 2020; Callan, 2020; Davis, 2020; Grobbelaar et al., } \\
\text { 2017; Gregorova y Heinzova, 2019; Guignona et al., 2021; Co- } \\
\text { rretgé y Miret, 2018; Lawton, 2019; Maistry y Lortan, 2017; } \\
\text { - Mercer-Mapstone y Bovill, 2020; Mitchell et al., 2019; Olson, } \\
\text { 2019; Reilly, } 2018 .\end{array}$} \\
\hline & & Actualización del currículo. & \\
\hline & & Compromiso del profesor. & \\
\hline & & Compromiso del alumno. & \\
\hline & EXTU & Enseñanza-aprendizaje & Ossola, 2018; \\
\hline & \multirow{3}{*}{ CC } & Aprendizaje servicio & \multirow{3}{*}{$\begin{array}{l}\text { Alcantar, 2017; Davis, 2020; Goldner y Golan, 2019; } \\
\text { Karliani et al.,2019; Mitchell et al.,2019; Owusu-Agyeman y } \\
\text { Fourie-Malherbe, 2019; Palombaro et al., 2017; Reilly, } 2018 .\end{array}$} \\
\hline & & Voluntariado & \\
\hline & & Liderazgo social. & \\
\hline & \multirow{2}{*}{ CUC } & $\begin{array}{l}\text { Educación para el desarrollo } \\
\text { sostenible. }\end{array}$ & \multirow{2}{*}{$\begin{array}{l}\text { Callan, 2020; Davis, 2020; Dirksen, 2020; Dulaney, 2018; Ex- } \\
\text { ner et al., 2017; Grobbelaar et al., 2017; Joseph y Carolissen, } \\
\text { 2019; Lawton, 2019; Maistry y Lortan, 2017; Mercer-Mapstone } \\
\quad \text { y Bovill, 2020; Quillinan et al., 2018. }\end{array}$} \\
\hline & & $\begin{array}{l}\text { Creación colaborativa del co- } \\
\text { nocimiento. }\end{array}$ & \\
\hline
\end{tabular}

Promoción de valores democráticos, cívicos y sociales (ciudadanía, participación, justicia social, solidaridad, igual-

RSU

Gestión socialmente responsable. Investigación pertinente.
Gómez et al., 2018; González-Pérez et al., 2021; Latif, 2018; Lima-Ravelo et al., 2018; Martínez-Buján et al., 2020; Menegat et al., 2018; Vallaeys y Álvarez, 2019; Vallaeys, 2021. sión)

\section{Promoción del aprendi-} zaje a lo largo de la vida.

Promoción de habilidades para mejorar la empleabilidad.
CUC Enseñanza-aprendizaje
(aprendizaje para adulto y
educación informal). Enseñanza-aprendizaje adeCUC y RSU cuando el currículo a la demanda del mercado.
Douglas et al., 2020.

Dulaney, 2018.

\section{| Discusión y conclusiones}

La presente revisión sistemática confirma la tendencia en la educación superior que "redescubre" en la relación con el entorno, un apoyo necesario, para conseguir las transformaciones sociales y recuperar la dimensión colectiva en la construcción del bien común en la sociedad (Preece,2016).

De acuerdo con la UNESCO (2015), "el bien común está constituido por los bienes que los seres humanos comparten intrínsecamente en común y que se comunican entre sí, como los valores, las virtudes cívicas y el sentido de la justicia" (p.85). Esto implica construir relaciones socioeducativas sobre la base de la solidaridad, el diálogo y la participación colectiva. Este trabajo ha ilustrado cómo las IES desde diferentes contextos geográficos y culturales han "contextualizado" e interpretado estos postulados. Por esta razón, se observa una convergencia de finalidades y estrategias entre las distintas orientaciones señaladas en la tabla 2, lo cual se podría atribuir a esta gran finalidad compartida por la educación superior a nivel global, "la construcción del bien común en la sociedad" que ha sido aceptada por todos los países y ha sido expresada en los Objetivos de Desarrollo Sostenible de la Agenda 2030.

Asimismo, a partir de la presente revisión se observa que las diferentes orientaciones identificadas están relacionadas 
con la demanda de los diferentes entornos. Así los términos EXTU, RSU, construidos desde Latinoamérica, se fundamenta en la solidaridad, característica de las IES frente a la desigualdad de la región. Varias iniciativas han buscado y buscan promover un diálogo solidario y responsable para superar la desigualdad del continente. Por ejemplo, el proyecto Universidad construye País, surgido en Chile en el 2001; la Asociación de Universidades de la Compañía de Jesús en América Latina (AUSJAL); y la Red de Unión de Responsabilidad Social de Latinoamérica (URUSULA), son solo algunas de las diferentes iniciativas implementadas en la interacción con el entorno (Gaete, 2018, 2020; Gaete y Álvarez, 2019; Gómez et al., 2018; González-Pérez et al., 2021; Grobbelaar et al., 2017; HuertaRiveros y Gaete-Feres, 2017; Vallaeys, 2021).

Mientras el CUC y CC, son orientaciones más centradas en el diálogo con la comunidad y la participación política, arraigadas en las IES de EE.UU., como respuesta al problema del racismo sistémico que atenta contra la democracia y la convivencia social. La red Campus Compact, es la única asociación nacional de educación superior estadounidense, cuyo principio fundamental es la defensa de la democracia a través del compromiso cívico y comunitario (Alcantar, 2017; Callan, 2020; Davis, 2020; Dirksen, 2020; Palombaro et al., 2017; Reilly, 2018). Asimismo, el CUC, ha tenido una gran receptividad en Sudáfrica por la herencia del apartheid, la pobreza, el escaso desarrollo socioeconómico y humano y la creciente carga sanitaria, obligando a las IES a desarrollar una mayor sensibilidad social reconsiderando cómo la enseñanza, y la investigación, pueden favorecer el compromiso con la comunidad, para transformar las sociedades (Bhagwan, 2018, 2020; Exner et al., 2017; Grobbelaar et al., 2017; Joseph y Carolissen, 2019; Mbah et al.,2021).

Los resultados muestran que la tipología desarrollada por Hollister (2017) se ha ampliado, especialmente en Europa donde conviven diferentes orientaciones. La "Renovada
Agenda para la educación superior" es un claro ejemplo, al proponer una mayor conexión de la educación superior con la sociedad por medio de dos propuestas. Primero, con la investigación a través de la iniciativa "Horizonte 2020", que desarrolla el programa Ciencia con y para la Sociedad (por su acrónimo en inglés SwafS), que consiste en acercar la ciencia a la ciudadanía y se caracteriza por el impulso de la investigación comprometida ER, que comienza a expandirse a África (Mbah et al.,2021); Segundo, con la invitación a promover una mayor participación de la comunidad en las IES a través del CUC, con el proyecto "Towards an European Framework for Community Engagement in Higher Education" TEFCE.

En este contexto, las diversas orientaciones halladas RSU, CUC, CC, EXTU y ER, resaltan el valor del entorno para que las IES cumplan asertivamente su rol en la sociedad. Quizás en el futuro, sea posible a partir de "la contextualización", construir un "ecosistema de educación superior más integrado", con una interrelación más flexible que dinamice mucho más la relación de IES con el entorno. Para ello, es necesario seguir profundizando el autodiagnóstico y la evaluación, como camino a la integración de los propósitos reproductivos-económicos y los propósitos sociales-humanizadores de la educación superior (Ashwin, 2020).

De igual forma, desde esta revisión sistemática se afirma que la RSU se ha consolidado como un concepto aglutinante y transformador que revitaliza la función social de las IES en diferentes contextos geográficos y culturales (Appe, et al. 2017; Latif, 2018; Lima-Ravelo et al., 2018; MartínezBuján et al., 2020; Shek \& Hollister, 2017). Para Vallaeys y Álvarez (2019), esto se debe a que la RSU posee una doble dimensión. Por un lado, está la gestión ética y eficiente de los recursos e incluye todos los procesos administrativos, y, por otro lado está la participación social, relacionada con las funciones sustantivas de las IES. Mientras que otras orientaciones CUC, CC, EXTU y ER, ponderan más 
la dimensión de participación social (Benneworht et al. 2018; Hoyt, 2014; Preece, 2016), dejando algunos vacíos a nivel de la gestión institucional. Por ejemplo, Farnell et al., (2020); señalaron que el CUC refiere al desarrollo de asociaciones mutuamente beneficiosas entre las universidades y sus comunidades externas, con el fin de abordar las necesidades de la sociedad, y es, por tanto, "un" aspecto fundamental de la responsabilidad social de la educación superior. A pesar de estos matices, es claro que todas las orientaciones sobre la interacción de las IES con el entorno ponen en el centro de su misión valores como: respeto, igualdad, y solidaridad.

En tal sentido, se espera que el diálogo de las IES con el entorno, transversalicen e integren los procesos de aprendizaje, el desarrollo de la identidad personal, y la convivencia social (Engeström y Käyhkö, 2021), en el establecimiento de un nuevo contrato social más integral, incluyendo a gobiernos, empresas, sociedad y naturaleza para logar una sociedad más justa, solidaria y sostenible (Vallaeys, 2021). En otras palabras, se espera que las IES susciten una interdependencia con impactos positivos a nivel personal y colectivo, en el horizonte del bien común, expresados en comportamientos ciudadanos con criterios éticos y responsables

Finalmente, y para concluir, se sugiere que la agenda de investigación futura promueva la realización de estudios con una perspectiva comparada entre las diferentes actividades implementadas por las IES, para socializar y aprender mutuamente de las capacidades desarrolladas en los diversos entonos. Igualmente, se podrían desarrollar trabajos que relacionen el empleo de nuevas pedagogías en la interacción de las IES con el entorno, como las comunidades de prácticas, aprendizajes basados en proyectos sociales, o fomentar más la investigación comprometida, y potenciar la gestión social del conocimiento a través de la actualización del currículo con aportes de diferentes actores sociales.

\section{| Notas}

i Este artículo forma parte de una investigación más amplia sobre el "compromiso universidad-comunidad" y las redes universitarias internacionales, gracias a una beca de formación de la Cátedra Unesco-Santander de la Universidad de Deusto.

\section{| Referencias}

Ahumada-Tello, E., Ravina-Ripoll, R., \& López-Regalado, M. (2018). Responsabilidad Social Universitaria. Desarrollo de competitividad organizacional desde el proceso educativo. Actualidades Investigativas en Educación, 18(3), 359-389. https://doi.org/h9rj

Alcantar, C. (2017). Cultivating Our Nation's Engaged Citizenry: Institutional Factors That Promote the Civic Engagement of College Students. University of California. https://eric.ed.gov/?id=ED580604

Appe, S., Rubaii, N., Líppez-De Castro, S., \& Capobianco, S. (2017). The Concept and Context of the Engaged University in the Global South: Lessons from Latin America to Guide a Research Agenda. Journal of Higher Education Outreach and Engagement, 21(2), 7-36.

Ashwin, P. (2020). Transforming university education. A manifestó. Bloosbury Academic.

Bakar, F., Sharif, Z., \& Abdullah, Z. (2019). Managing University-Community Engagement (UCE): The Case of UUM. International Journal of Innovative Technology and Exploring Engineering (IJITEE), 8, 199-205.

Balázs, L., Rajcsányi-Molnár, M., András, I., \& Sitku, K. (2021). Social Responsibility and Community Engagement at a Hungarian Regional University. Journal of Higher Education Theory and Practice, 21(1), 53-62. https://doi.org/h9rm

Barreno, M., Barreno, Z., \& Olmedo, A. (2018). La educación superior y su vinculación con la sociedad: referentes esenciales para un cambio. Revista Universidad y Sociedad, 10(3), 40-45. 
Benneworth, P., Culum, B., Farnell, T., Kaiser, F., Seeber, M., Scukanec, N. Westerheijden, D. (2018). Mapping and Critical Synthesis of Current State-of-the-Art on Community Engagement in Higher Education. Institute for the Development of Education. Zagreb: Institute for the Development of Education TEFCE. https://cutt.ly/ sX66EIw

Bhagwan, R. (2018). University-community Partnerships: Demystifying the Process of Engagement. South African Review of Sociology, 49 (3-4), 32-54. https://doi.org/ $\underline{\text { h9rn }}$

Bhagwan, R. (2020). Student volunteer experiences as a way to advance teaching and learning: a call for community service. The Independent Journal of Teaching and Learning, 15(2), 8-23.

Callan, M. (2020). An Examination of Benefit and Equity in Community-University Service-Learning Partnerships. Virginia: The College of Saint Mary. https:// cutt.ly/YCqtAMQ

Coelho, M., \& Menezes, I. (2020). University social responsibility as a driving force of change: students' perceptions beyond the ivory tower. On the Horizon, 28(2), 93-100. https://doi.org/h9rv

Compagnucci, L., \& Spigarelli, F. (2020). The Third Mission of the university: A systematic literature review on potentials and constraints. Technological Forecasting and Social Change, 161, 1-30. https://doi.org/gkbkgp

Corretgé, R., \& Miret, J. (2018). Adaptación de los estándares GRI y creación de indicadores de RSU: Un trabajo conjunto de la Universitat de Barcelona y la Universitat Pompeu Fabra. Revista Digital de Investigación en Docencia Universitaria, 12(1), 137-155. https://doi.org/ $\underline{\mathrm{h} 9 \mathrm{r} 2}$

Cox, J. (2018). Beyond Objectivity: Examining the Effects of Incorporating Civic Engagement into Higher Education Journalism Courses. Journal of Community Engagement and Higher Education, 10(2), 22-29.

Davis, M. (2020). Civic Engagement of Campus-Based and Online Undergraduate Students: A Quantitative Causal-Comparative Study (Tesis doctoral, Grand
Canyon University). Repositorio Proquest . https:// cutt.ly/2T8SZ4C

De Ketele, J. M. (2008). La pertinencia social de la educación: Colaboración especial I. 6. En GUNI (ed). La educación superior en el mundo: Educacion superior, Nuevos retos y roles emergentes para el desarrollo humano y social (pp. 55-61). GUNI. Ediciones Mundi-Prensa.

Dirksen, C. (2020). Community engagement for student faith development: Service-learning in the Pentecostal tradition. Christian Higher Education, 19(1-2), 78-90. https://doi.org/h9r3

Douglas, J., Kilpatrick, S., Katersky-Barnes, R., Alderson, R., \& Flittner, N. (2020). Embedding tertiary education in rural communities: building 'warm connections'. Studies in Continuing Education, 42(1), 61-74. https:// doi.org/ghd9hr

Dulaney, T. (2018). How Can Clarion University of Pennsylvania Support the Region, Employers, and Students in Order to Meet Emerging and Changing Workforce Needs in the Petrochemical and Plastics Industries (Tesis doctoral, Universidad de Pittsburgh). Repositorio Proquest https://cutt.ly/AT8S2KS

Engeström, R., \& Käyhkö, L. (2021). A critical search for the learning object across school and out-of-school contexts: A case of entrepreneurship education. Journal of the Learning Sciences, 30 (3), https://doi.org/gqpdmt Esteruelas, N. (2018). Responsabilidad social universitaria: el papel de los medios de comunicación y sus stakeholders. Retos: Revista de Ciencias de la Administración y Economía, 8 (16), 95-105. https://doi.org/h9r4

Exner, R., Jansen, J., Stroud, L., \& du Preez, M. (2017). A multidisciplinary approach to university engagement: key considerations for dynamic mental health teaching and service provision to a disadvantaged community. Educational Research for Social Change, 6(2). 6075. https://doi.org/h9r5

Farnell, T., Ćulum, B., Dusi, D., O’Brien, E., Šćukanec, N., Veidemane, A., \& Westerheijden, D. (2020). Community engagement in higher education: trends, practices and policies. NESET report. Publications Office of the Eu- 
ropean Union. https://cutt.ly/KT8DefG

Gaete, R. (2018). Conciliación trabajo-familia y responsabilidad Social Universitaria: Experiencias de mujeres en cargos directivos en universidades chilenas. Revista Digital de Investigación en Docencia Universitaria, 12(1), 81-100. https://doi.org/h9r6

Gaete, R. (2020). Dirección por valores y responsabilidad social en universidades estatales chilenas. Revista Digital de Investigación en Docencia Universitaria, 14(1). e1073. https://doi.org/h9r7

Gaete, R., \& Álvarez, J. (2019). Responsabilidad social universitaria en Latinoamérica. Los casos de URSULA y AUSJAL. Actualidades Investigativas en Educación, 19(3), 1-27. https://doi.org/h9r8

Goldner, L., \& Golan, D. (2019). What is meaningful civic engagement for students? Recollections of Jewish and Palestinian graduates in Israel. Studies in Higher Education, 44(11), 1945-1959. https://doi.org/gfwpjm

Gómez, L., Alvarado, Y., \& Pujols, A. (2018). Practicando la Responsabilidad Social Universitaria en el Caribe: Perspectivas de los públicos internos. Revista digital de investigación en docencia universitaria, 12(1), 101120. https://doi.org/h6gb

González-Pérez, M., Cordova, M., Hermans, M., Nava-Aguirre, K., Monje-Cueto, F., Mingo, S., \& Floriani, D. (2021). Crises conducting stakeholder salience: shifts in the evolution of private universities' governance in Latin America. Corporate Governance, 21(6), 1194-1214. https://doi.org/h9r9

Gregorova, B., \& Heinzova, Z. (2019). Specifics of Measuring Social and Personal Responsibility of University Students After Completion of a Service-Learning Course in Slovak Conditions. Journal of Higher Education Outreach and Engagement, 23(3), 104-111.

Grobbelaar, H., Napier, C., \& Maistry, S. (2017). Reinforcing paternalism? The need for a social justice approach to prepare students for community engagement at universities of technology. Journal for New Generation Sciences, 15(1), 74-93. https://cutt.ly/kCqxMWx

Guignona, M., Halili, S., Cristobal, F., Woolley, T., Reeve, C.,
Ross, S., \& Neusy, A. (2021). A Curriculum for Achieving Universal Health Care: A Case Study of Ateneo de Zamboanga University School of Medicine. Frontiers in Public Health, 9, 612035. https://doi.org/h9sc

Hollister, R. (2017). Theoretical framework and global institutional experience En D. Shek, \& R. Hollister (Eds),University Social Responsibility and Quality of Life. A Global Survey of Concepts and Experiences (pp. 10-15). Springer.

Hoyt, L. (2014). University Civic Engagement: A Global Perspective. Kettering Fundación. En D. Brown, D. Witte, \& J. Easton (Eds), Higher Education Exchange (pp. 42-52). Kettering Foundation. https://cutt.ly/rCqvJ2C Huerta-Riveros, P., \& Gaete-Feres, H. (2017). Responsabilidad social universitaria a través de los reportes de sostenibilidad del Global Reporting Initiative: experiencia de una universidad pública. Revista Iberoamericana de Educación Superior, 8(23), 120-137.

Huberman, A. M., \& Miles, M. B. (1994). Data management and analysis methods. In N. K. Denzin \& Y. S. Lincoln (Eds.), Handbook of qualitative research (pp. 428-444). Thousand Oaks (CA): Sage Publications.

Jie, C., \& Huam, H. (2019). Predictors of reputation through university social responsibility practices in a Malaysian private university: The customer's perspective. Journal Komunikasi: Malaysian Journal of Communication. 35(3), 316-333. https://doi.org/h9sd

Jiménez, Á., \& Aldana, E. (2020). Revisión sistemática exploratoria: una perspectiva de la Educación Superior a distancia desde la investigación histórica. Revista Espacios, 41(4), 1-8.

Jiménez, J., Maiztegui-Oñate, C. y Da Silva Pinto, J. L (2021). La investigación en las redes universitarias internacionales: una mirada desde el compromiso universidad-comunidad. En Márquez, C. (dir.) (2021). Transformación universitaria. Retos y oportunidades. (pp. 465-476). Ediciones Universidad de Salamanca.

Joseph, B., \& Carolissen, R. (2019). Citizenship: A core motive for South African university student volunteers. Education, Citizenship and Social Justice, 14(3), 225-240. 
Interacción de las Instituciones de Educación Superior con el entorno: Orientaciones, Finalidades y Estrategias. Una revisión sistemática de la literatura, 2017-2021

\section{https://doi.org/gfpf2g}

Karasik, R. (2020). Community Partners' Perspectives and the Faculty Role in Community-Based Learning. Journal of Experiential Education, 43(2), 113-135. https:// doi.org/gmc2xp

Karliani, E., Kartadinata, S., Winataputra, U., \& Komalasari, K. (2019). Indonesian civic engagement among college students. Journal of Human Behavior in the Social Environment, 29(5), 582-592. https://doi.org/h9sf

Latif, K. (2018). The development and validation of stakeholder-based scale for measuring university social responsibility (USR). Social indicators research, 140, 511-547. https://doi.org/gfksvk

Lawton, P. (2019). At the crossroads of intersecting ideologies: Community-based art education, community engagement, and social practice art. Studies in Art Education, 60, 203-218. https://doi.org/gjftzj

Lima-Ravelo, W., Cabra-Copete, M., \& Tascón-Hoyos, D. (2018). Medición del impacto de Responsabilidad Social Universitaria. Caso Universidad Santo Tomás, Colombia. Opción, 34, 935-963.

Lo, C., Xue Pang, R., \& Hon-Ying Li, P. (2017). University social responsibility: conceptualization and an assessment framework . En D. Shek, \& R. Hollister, University Social Responsibility and Quality of Life, Quality of Life. (pp.37-59, Springer.

Locatelli, R. (2018). La educación como bien público y común: reformulación de la gobernanza de la educación en un contexto cambiante. Documentos de trabajo de investigación y prospectiva en educación de la UNESCO. UNESCO. https://cutt.ly/xCqmPuF

Maistry, S., \& Lortan, D. (2017). Lessons from the global South: Knowledge democracy and epistemic justice in higher education institutions in South Africa. Journal for New Generation Sciences, 15(1), 123-139. https://cutt.ly/hCqPVyY

Martínez-Buján, R., Santiago-Gómez, E., Diz, C., Cortes-Vázquez, J., \& Golías, M. (2020). Campus greening from social sciences: emerging formulas on social responsibility and teaching innovation. Internatio- nal Journal of Sustainability in Higher Education, 21(7), 1545-1561. https://doi.org/h9sk

Martínez-Usarralde, M., Gil-Salom, D., \& Macías-Mendoza, D. (2019). Revisión sistemática de Responsabilidad Social Universitaria y Aprendizaje Servicio. Análisis para su institucionalización. Revista mexicana de investigación educativa, 24(80), 149-172.

Mbah, M. (2019). Can local knowledge make the difference? Rethinking universities' community engagement and prospect for sustainable community development. The Journal of Environmental Education, 50, 11-22. https://doi.org/h9sm

Mbah, M., Johnson, A., \& Chipindi, F. (2021). Institutionalizing the intangible through research and engagement: Indigenous knowledge and higher education for sustainable development in Zambia. International Journal of Educational Development, 82, 102371. https://doi.org/h9sn

Medina, R., Franco, M., Torres, L., Velázquez, K., Valencia, M., \& Valencia, A. (2017). La responsabilidad social universitaria en la actual sociedad del conocimiento. Un acercamiento necesario. MediSur, 15(6), 786-791.

Menegat, J., Sarmento, D., \& Calderón, A. (2018). Princípios de responsabilidade social na Educação Superior: tendências na gestão de instituições confessionais. Revista Digital de Investigación en Docencia Universitaria, 12(1), 59-80. https://doi.org/h9sp

Mercer-Mapstone, L., \& Bovill, C. (2020). Equity and diversity in institutional approaches to student-staff partnership schemes in higher education. Studies in Higher Education, 45, 2541-2557. https://doi.org/ ghw5mf

Mitchell, T., Rost-Banik, C., \& Battistoni, R. (2019). Civic agency and political engagement: Community engagement's enduring influence. Journal of Student Affairs Research and Practice, 56(5), 582-594. https:// doi.org/h9sq

Newman, M., \& Gough, D. (2020). Systematic Reviews in Educational Research: methodology, perspectives and application. En O. Zawacki-Richter, M. Kerres, S. 
Bedenlier, M. Bond, \& K. Buntins (Eds,), Systematic Reviews in Educational Research (pp.3-22). Springer.

Olson, B. (2019). Higher Education and Community Development: A Multi-Stakeholder View on the Correlates of Project Participation and Design Preferences (Tesis doctoral, Pennsylvania State University). https://cutt.ly/lCqSMO9

Organización de las Naciones Unidas para la Educación, la Ciencia y la Cultura [UNESCO]. (1998). La educación superior en el siglo XXI: visión y acción. Conferencia Mundial sobre la Educación Superior. Educación superior y sociedad, 9(2), 97-113. https://cutt.ly/tT8Dij8

Organización de las Naciones Unidas para la Educación, la Ciencia y la Cultura [UNESCO]. (2015). Replantear la educación ¿hacia un bien común mundial? Ediciones UNESCO. https://cutt.ly/8T8DdqD

Organización de las Naciones Unidas para la Educación, la Ciencia y la Cultura [UNESCO]. (2021). Pensar más allá de los límites: Perspectivas sobre el futuro de la educación superior para 2050. Ediciones UNESCO

Ossola, M. (2018). Educación Superior y diversidad cultural. Análisis de las experiencias formativas de jóvenes indígenas en proyectos de extensión universitaria. Praxis Educativa, 22(3), 56-63. https://doi.org/h9sr

Owusu-Agyeman, Y., \& Fourie-Malherbe, M. (2019). Students as partners in the promotion of civic engagement in higher education. Studies in Higher Education, 46 (6), 1241-1255. https://doi.org/h9ss

Palombaro, K., Black, J., Dole, R., Pierce, J., Santiago, M., \& Sabara, E. (2017). Assessing the Development of Civic Mindedness in a Cohort of Physical Therapy Students. Journal of the Scholarship of Teaching and Learning, 17(4), 31-43. https://doi.org/h9st

Preece, J. (2016). The Porous University: re-thinking community engagement. Alternation, 23(1), 208-232.

Prisma Group . (2009). Preferred Reporting Items for Systematic Reviews and Meta-Analyses. The Prisma Statement. Quillinan, B., McEvoy, E., MacPhail, A., \& Dempsey, C. (2018). Lessons learned from a community engagement initiative within Irish higher education. Irish
Educational Studies, 37(1), 113-126. https://doi.org/gfvp4z

Reilly, J. (2018). A Qualitative Case Study of Engagement in Higher Education: The Influence of Teachers' Experiences on Classroom Engagement at a Southern State University (Tesis doctoral, Northcentral University). https://cutt.ly/5T8Dh9V

Roy, R., \& El Marsafawy, H. (2021). Bridging Recognition of Prior Learning (RPL) and Corporate Social Responsibility (CSR): Circular Flow of Interaction among the University, Industry, and People. Sustainability, 13(8), 2-25. https://doi.org/h9sv

Ruiz-Bejarano, A., \& Alastor, E. (2018). La organización de jornadas en el aula académica: Innovación docente y Compromiso Social Universitario. Revista Digital de Investigación en Docencia Universitaria, 12(1), 176-188. https://doi.org/h9sw

Salcedo-Muñoz, V., Nuñez, L., Campuzano, J., Lucas, J., \& Nuñez, W. (2017). Enfoque exploratorio sobre acciones de Responsabilidad Social en universidades del Ecuador: Caso UPSE y UTMACH. Espacios, 38(35), 3835.

Schnurbus, V., \& Edvardsson, R. (2020). The Third Mission Among Nordic Universities: A Systematic Literature Review. Scandinavian Journal of Educational Research. 1-23. https://doi.org/h9sx

Shek, D., Ma, C., \& Yang, Z. (2020). Transformation and development of university students through service-learning: A corporate-community-university partnership initiative in Hong Kong (Project WeCan). Applied Research in Quality of Life, 15, 1375-1393. https://doi.org/h9sz

Shek, D., Yuen-Tsang, A., \& Eddie, C. (2017). USR network: a platform to promote university social responsibility. En D. Shek, \& R. Hollister, University Social Responsibility and Quality of Life. A Global Survey of Concepts and Experiences (pp.11-24). Springer.

Vallaeys, F. (2021). Manual de Responsabilidad Social Universitaria. El modelo URSULA: estrategias, herramientas, indicadores. Ed. URSULA. 
Vallaeys, F., \& Álvarez, J. (2019). Hacia una definición Latinoamericana de Responsabilidad Social Universitaria. Aproximación a las preferencias conceptuales de los universitarios. Educación XXI, 93-116. https:// doi.org/h6gg

Vásquez, V. (2019). La Responsabilidad Social en la Educación Superior: Una revisión de la alineación entre el discurso educativo oficial y el curriculum explícito de las instituciones de educación superior de Oaxaca. Revista de la educación superior, 48(191), 115-139.

Unión Europea [UE]. COM/2017/164 final. Comunicación de la comisión al parlamento europeo, al consejo, al comité económico y social europeo y al comité de las regiones sobre una agenda renovada de la UE para la educación superior. https://cutt.ly/dCqGMu4

Yampufé, J., Antezana, S., Najarro, J., \& Oriondo, R. (2021). Línea de base de la responsabilidad social en una universidad pública: eje de participación social. Dilemas contemporáneos: educación, política y valores, 8(SPE2)). https://doi.org/hw4r 